\title{
RECURSIVE ALGORITHMS FOR THE ELIMINATION OF REDUNDANT PATHS IN SPATIAL LAG OPERATORS*
}

\author{
Hans J. Blommestein \\ OECD, 2 rue André-Pascal 75755 Paris Cedex 16, France and University of Twente, P.O. \\ Box 217, 7500 AE Enschede, The Netherlands
}

Nick A. M. Koper

Philips Semiconductors, Innovation and Engineering Group, P.O. Box 10,9500 AA

Stadskanaal, The Netherlands

\begin{abstract}
Recursive algorithms for the elimination of redundant paths in spatial lag operators are introduced. It is shown that these algorithms have superior computational properties in comparison with the cumbersome procedure proposed by Ross and Harary (1952). A rigorous definition of spatial lag operators is given, while a number of mathematical results and properties are derived. Theoretical and empirical results regarding the performance of the proposed algorithms are presented.
\end{abstract}

\section{INTRODUCTION}

In this article we study the problem of redundant paths in spatial lag operators. Recursive algorithms for the elimination of redundant paths are presented. It is shown that these algorithms have superior computational properties in comparison with the cumbersome, non-recursive procedure proposed by Ross and Harary (1952).

Basic mathematical concepts are introduced in Section 2. The adverse consequences of redundant paths in spatial lag operators for the estimation of spatial econometric models are illustrated in Section 3. Next, we introduce in Section 4 recursive algorithms for the elimination of redundant paths. In Section 5 we study the complexity and performance of the various algorithms in both theoretical and empirical terms. Conclusions are summarized in the final Section 6 .

The appendices contain details on Ross and Harary's algorithm (Appendix A) and Blommestein and Koper's algorithms (Appendices B, C and D).

\section{BASIC MATHEMATICAL CONCEPTS}

In spatial economics, international economics, geography, biometrics and other fields where the element of space plays an important role, the spatial lag operator $\mathbf{L}_{S}$ is a useful tool for the quantitative analysis of a wide range of problems.

*The authors are indebted to the excellent suggestions and comments by the editor and two anonymous referees on a previous draft of this paper. We are of course responsible for remaining errors.

Received August 1990; revised January 1991; accepted August 1991. 
Definition 1 (Spatial Lag Operator). Let $\mathscr{V}_{R}$ be a $R$-dimensional vector space over a field $\mathscr{F}, \mathbf{K}=\left\{x_{1}, x_{2}, \ldots, x_{R}\right\}$ any basis of $\mathscr{V}_{R}$, and $\mathbf{L}_{S}$ a linear transformation of $\mathscr{V}_{R}$ into itself, i.e., $\mathbf{L}_{S}: \mathscr{V}_{R} \rightarrow \mathscr{V}_{R}$. Since every vector is a linear combination of the basis vectors $\left\{\mathbf{x}_{r} \mid r=1,2, \ldots, R\right\}$ we have

$$
\mathbf{L}_{\mathrm{S}} \mathbf{x}_{r}=\sum_{r=1}^{R} w_{r r^{\prime}} \mathbf{x}_{r} \quad\left(\text { for } r^{\prime} \in\{1,2, \ldots, R\}\right)
$$

Once the ordered basis $\mathbf{K}$ is fixed, any linear transformation is completely and uniquely determined by the set with scalars $\left\{w_{r r^{\prime}} \mid r, r^{\prime}=1,2, \ldots, R\right\}$ in $\mathscr{F}$. The set $\left\{w_{r r}\right\}$ of $R^{2}$ scalars defines the spatial lag operator $\mathbf{L}_{S}$

$$
\mathbf{L}_{S}=\left[\begin{array}{llllll}
w_{11} & w_{12} & \cdot & \cdot & \cdot & w_{1 R} \\
w_{21} & w_{22} & \cdot & \cdot & \cdot & \cdot \\
\cdot & & \cdot & & & \cdot \\
\cdot & & & \cdot & & \cdot \\
\cdot & & & & \cdot & \cdot \\
w_{R 1} & w_{R 2} & \cdot & \cdot & \cdot & w_{R R}
\end{array}\right]
$$

Definition 1 is very general. In most applications it will be necessary to impose additional restrictions on $\mathbf{L}_{S}$. These restrictions may differ across applications.

(a) The real $(R \times R)$ matrix $\mathbf{L}_{S}=\left[w_{r r^{\prime}}\right]$ is non-negative, i.e., $w_{r r^{\prime}} \geq 0, \forall r \neq r^{\prime} \in$ $\{1,2, \ldots, R\}$; it is further assumed that $w_{r r}=0, \forall r \in\{1,2, \ldots, R\}$. In some cases we require that the spatial lag operator is row stochastic. For example, in spatial econometric applications (see Example 2 in Section 3). An $(R \times R)$ matrix $\mathbf{L}_{S}$ is row stochastic when $\mathbf{L}_{S} \geq 0$ and

$$
\sum_{r^{\prime}=1}^{R} w_{r r^{\prime}}=1 \quad(\forall r \in\{1,2, \ldots, R\})
$$

(b) the (non-)boolean $(R \times R)$ matrix $\mathbf{L}_{S}$ has one of the three general mathematical properties below

(1) $\mathbf{L}_{S}$ is a real $(R \times R)$ symmetrix matrix. That is: $\mathbf{L}_{S}=\mathbf{L}_{S}^{T}$;

(2) $\mathbf{L}_{S}$ is a real $(R \times R)$ asymmetric matrix, which is non-triangular;

(3) $\mathbf{L}_{S}$ is a real $(R \times R)$ upper or lower triangular matrix. That is: $w_{r r^{\prime}}=$ $0, \forall r>r^{\prime} \in\{1,2, \ldots, R\}$ for upper triangular and $w_{r r^{\prime}}=0, \forall r<r^{\prime} \in$ $\{1,2, \ldots, R\}$ for lower triangular matrices.

Remark 1. The temporal lag operator L (see Dhrymes, 1981) can be written as a square matrix in triangular form, that is, as a matrix containing zeros apart from ones on the first upper diagonal.

The spatial lag operator from Definition 1 can be used in the construction of many different types of space(-time) models (for an overview see Blommestein, $1985 \mathrm{~b}$ ). A common characteristic of these models is that they constitute a multivariate system which exhibits systematic dependence between the observa- 
tions at each spatial unit (regions, towns, countries, etc.) and the observations at neighboring spatial units (= spatial autocorrelation). The operator $\mathbf{L}_{S}$ is a very parsimonious way of expressing spatial order in this multivariate system.

Let $\left\{w_{r r^{\prime}}^{p}\right\}$ be the elements of $\mathbf{L}_{S}^{p}\left(\mathbf{L}_{S}\right.$ raised to the power $\left.p\right)$. Then, $\left\{w_{r r^{\prime}}^{p}\right\}$ are nonzero if the spatial units $r$ and $r^{\prime}$ are $p$ th order neighbors. The exact specification of the form of $\left\{w_{r r}^{p}\right\}$ is dependent on the spatial (economic) phenomena to be studied. A very general way of interpreting the information conveyed by $\mathbf{L}_{S}^{p}$ is to construct the incidence matrix $\mathbf{Q}$, corresponding to $\mathbf{L}_{S}$. Next, consider $\mathbf{Q}$ as a network representation of a spatial system consisting of $R$ spatial units. The non-zero entries of the $(R \times R)$ matrix $\mathbf{Q}$ correspond to the vertices, or nodes, of that network.

Proposition. If $\mathbf{Q}$ is raised to a positive power ( $p \geq 2)$, then the off-diagonal elements $\left(r \neq r^{\prime}\right)$ of $\mathbf{Q}^{p}$ denote the total number of connecting paths (or, equivalently, chains) between each pair of vertices $\left(x_{r}, x_{r^{\prime}}\right)$.

Proof. Note first that each element of the network $\mathbf{Q}, q_{r r^{\prime}}$ is either zero (i.e., absence of node) or one (i.e., node is present). Raise $\mathbf{Q}$ to the $p$ th power $(p \geqq 2)$. The typical elements $q_{r r^{\prime}}^{p}$ from $\mathbf{Q}^{p}$ can be written as

$$
q_{r r^{\prime}}^{p}=\sum_{k=1}^{R} q_{r k} q_{k r^{\prime}}^{p-1}
$$

This recursively defined summation gives then the total number of $p$-step connecting paths for each pair of vertices.

Remark 2. The entries $q_{r r}^{p}(r \in\{1,2, \ldots, R\})$ represent the number of $p$-step connecting paths of vertex $x_{r}$ with itself.

Now we are in a position to state the two central definitions of this article.

Definition 2a. Let $r, r_{1}, r_{2}, \ldots, r^{\prime}$ be arbitrary indices from the index set $\{1,2, \ldots, R\}$ of matrix $\mathbf{L}_{S}$. A sequence $\left(r, r_{1}, r_{2}, \ldots, r_{p-1}, r^{\prime}\right)$, for $p \geq 1$ and $r_{0}=r$, from the index set $\{1,2, \ldots, R\}$, is said to form a $p$-step path (or, equivalently, a chain of length $p)$ between the vertices or ordered pair $\left(x_{r}, x_{\mathrm{r}^{\prime}}\right)$ if $w_{r r_{1}} \cdot w_{r_{1} r_{2}} \cdot \ldots$. $w_{r_{p-2} r_{p-1}} \cdot w_{r_{p-1} r} \cdot>0$.

Definition 2b. A redundant path or chain (or, equivalently, circular route) is a path in which at least one index number repeats in the sequence of indices.

Remark 3. Raising (real) symmetric and asymmetric non-triangular $(R \times R)$ matrices $\mathbf{L}_{S}$ to powers of order $p>1$, will in general result in redundant paths, while this is not the case for triangular $\mathbf{L}_{S}$ (Blommestein, 1985a).

Definition $2 \mathrm{~b}$ can be made more transparent by considering the following example.

Example 1. Consider a system of six economic agents $\left(A_{1}, A_{2}, \ldots, A_{6}\right)$. Each agent can trade goods in its possession with other agents. Assume further that for institutional or economical reasons every agent can only trade directly with no more than three other agents. 
Consider economic agent $1\left(A_{1}\right)$. $A_{1}$ decides to sell goods to $A_{2}, A_{4}$ and $A_{5}$. Next consider economic agent $4\left(A_{4}\right) . A_{4}$ sells goods to $A_{1}, A_{3}$ and $A_{5}$. From Figure 1 it can be seen that $A_{1}$ cannot sell goods directly to $A_{3}$, but it is possible through $A_{4}$. Hence through an intermediate trader (i.e., in the second order) $A_{1}$ can sell goods to $A_{3}$ $\left(A_{1} \rightarrow A_{4} \rightarrow A_{3}\right)$. Suppose that we wish to exclude the possibility that goods sold by $A_{1}$ are sold back to $A_{1}$. If this would occur, we say that the goods followed a circular route or redundant path. For example, $A_{1} \rightarrow A_{4} \rightarrow A_{1}$ is a redundant path (of order 2).

In many cases these trading routes have to be ruled out as nonsensical (not in all cases!) from an economic point of view, necessitating the elimination of such redundant paths. It should be noted that goods can be sold by economic agents in alternative ways. For example, in the first order $A_{1}$ can sell goods to $A_{5}$, but also in the second order: $A_{4} \rightarrow A_{5}$ and $A_{1} \rightarrow A_{4} \rightarrow A_{5}$. An analysis of alternative trading routes may involve the problem of finding the shortest way to transport goods from one agent to another. We do not consider this problem here.

In Figure 1 spatial relations are described by means of a graph. Relations can also be described by means of a matrix. The matrix $\mathbf{L}_{S}$ is called a boolean spatial lag operator.

$$
\mathbf{L}_{S}=\left[\begin{array}{llllll}
\mathbf{0} & \mathbf{1} & \mathbf{0} & \mathbf{1} & \mathbf{1} & \mathbf{0} \\
1 & 0 & 0 & 1 & 1 & 0 \\
0 & \mathbf{1} & 0 & 0 & 1 & 1 \\
\mathbf{1} & \mathbf{0} & \mathbf{1} & \mathbf{0} & \mathbf{1} & \mathbf{0} \\
1 & \mathbf{1} & 0 & 1 & 0 & 0 \\
0 & 1 & 1 & 0 & 1 & 0
\end{array}\right]
$$

The first and the fourth row of the boolean spatial lag operator (set in bold face) correspond to the graph of Figure 1 (for the sake of simplicity the other rows of the matrix are not reflected in Figure 1). In the first row of the matrix $\mathbf{L}_{S}$ it can be seen that $A_{1}$ sells goods to $A_{2}, A_{4}$ and $A_{5}$, and in the fourth row of the matrix it can be seen that $A_{4}$ sells goods to $A_{1}, A_{3}$ and $A_{5}$. The columns of the matrix indicate who can buy goods from whom. For example, $A_{6}$ can buy goods from $A_{3}$ only and $A_{5}$ can

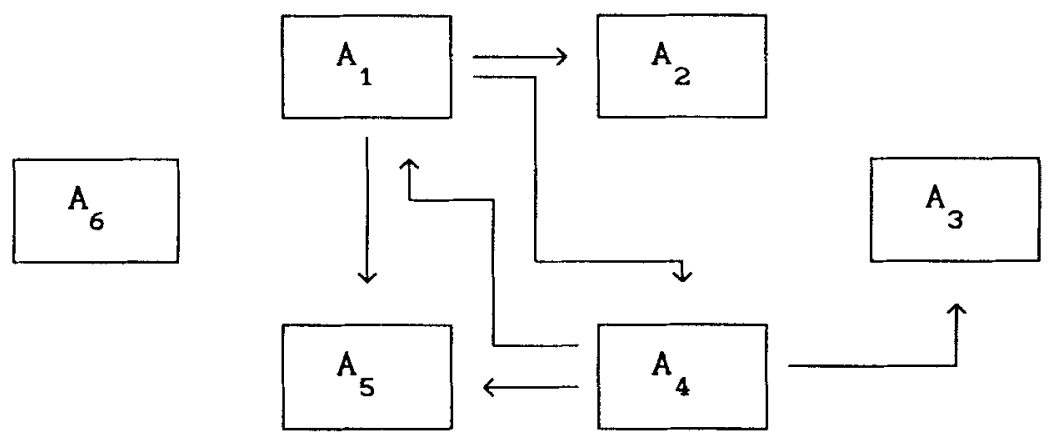

FIGURE 1: Spatial System with Six Economic Agents. 
buy goods from everybody except himself (that would be redundant in the first order).

The matrix $\mathbf{L}_{S}$ shows the number of one-step paths that goods have to travel from one economic agent to another (first-order paths), $\mathbf{L}_{S}^{2}\left(\mathbf{L}_{S}\right.$ squared) shows the number of two-step paths (second-order paths)

$$
\mathbf{L}_{S}^{2}=\left[\begin{array}{llllll}
3 & 1 & 1 & 2 & 2 & 0 \\
2 & 2 & 1 & 2 & 2 & 0 \\
2 & 2 & 1 & 2 & 2 & 0 \\
1 & 3 & 0 & 2 & 2 & 1 \\
2 & 1 & 1 & 2 & 3 & 0 \\
2 & 2 & 0 & 2 & 2 & 1
\end{array}\right]
$$

and $\mathbf{L}_{S}^{n}$ the number of $n$-step paths (paths of order $n$ ). An example of a third-order redundant path in $\mathbf{L}_{S}^{3}$ is: $A_{1} \rightarrow A_{5} \rightarrow A_{4} \rightarrow A_{1}$.

Also in many spatial econometric analyses the elimination of circular routes is required. As an example we briefly discuss in the next section the importance of eliminating circular routes in Simultaneous Spatial Autoregressive Distributed lag (SSAD) models.

\section{CONSEQUENCES OF REDUNDANT PATHS IN SIMULTANEOUS SPATIAL AUTOREGRESSIVE DISTRIBUTED LAG (SSAD) MODELS}

In Blommestein (1985b) the following linear SSAD model was studied:

$$
\mathbf{A Y}-\mathbf{X} \boldsymbol{\beta}=\mathbf{\epsilon}
$$

in which $\mathbf{A}=\mathbf{I}_{R}-\sum_{i=1}^{p} \alpha_{\mathrm{i}} \mathbf{L}_{S}^{i}$, a matrix of size $(R \times R) ; \mathbf{I}_{R}$ is an $(R \times R)$ identity matrix; $\mathbf{Y}$ is an $(R \times 1)$ vector with spatial observations on the dependent variable $y: \mathbf{X}$ is an $(R \times K)$ matrix with conditioning variables; $\left\{\alpha_{i} \mid i=1, \ldots, p ; \beta\right\}$ are parameters to be estimated; and $\epsilon \sim \operatorname{NID}\left(0, \sigma^{2} \mathbf{I}_{R}\right)$ is an $(R \times 1)$ vector with white noise error terms.

The log-likelihood corresponding to the SSAD model in (3.1) is equal to

$$
\mathscr{L}(\mathbf{Y})=(-R / 2) \cdot \ln \left(2 \pi \sigma^{2}\right)-\frac{1}{2} \sigma^{2}[\mathbf{A Y}-\mathbf{X} \beta]^{T}[\mathbf{A Y}-\mathbf{X} \beta]+\ln |\mathbf{A}|
$$

where $|\mathbf{A}|=\left|\mathbf{I}_{R}-\Sigma_{i=1}^{p} \alpha_{i} \mathbf{L}_{S}^{i}\right|$ is the Jacobian of the transformation from $\epsilon$ to $\mathbf{Y}$. It has been demonstrated in Blommestein (1985b) that the ML-estimations $\hat{\alpha}_{i}, \hat{\beta}$ and $\hat{\sigma}^{2}$ are contaminated by redundant information when the spatial lag operators $\mathbf{L}_{S}^{i}$ $(i=2, \ldots, p)$, contain circular routes (see Definition $2 \mathrm{~b}$ ). The next example shows that failure to eliminate circular routes might seriously distort estimated parameters.

Example 2. The following SSAD model in Blommestein (1985a) was used for an econometric analysis of some spatial features of the Irish economy:

$$
\mathbf{Y}=\delta_{0} \iota+\delta_{1} \mathbf{X}_{1}+\delta_{2} \mathbf{L}_{S}^{1} \mathbf{X}_{1}+\delta_{3} \mathbf{L}_{S}^{2} \mathbf{X}_{1}+\delta_{4} \mathbf{L}_{S}^{1} \mathbf{Y}+\delta_{5} \mathbf{L}_{S}^{2} \mathbf{Y}+\mathbf{E}
$$


where $\mathbf{Y}$ is an $(R \times 1)$ vector with observations on the percentage of gross agricultural output (in value terms) of each of the $R(=26)$ counties of Ireland consumed by the inhabitants of each county; $\mathbf{X}_{1}$ is an $(R \times 1)$ vector with values of an index of arterial road accessibility [see Cliff and Ord (1981) for details]; $\iota=$ $(1, \ldots, 1)^{T}$ of size $(R \times 1) ; \mathbf{L}_{\mathrm{S}}^{i}$ is a $i$ th order $(R \times R)$ spatial lag operator, where each non-zero off-diagonal element represents spatial interaction between a pair $(r$, $\left.r^{\prime}\right)$ of counties and all diagonal elements are zero; and $\mathbf{E} \sim \operatorname{NID}\left(0, \sigma_{e}^{2} \mathbf{I}_{R}\right)$ an $(R \times 1)$ vector with white noise error terms. Estimated parameters $\hat{\delta}=\left(\hat{\delta}_{0}^{e} \hat{\delta}_{1} \ldots \hat{\delta}_{5}\right)^{T}$ are given in Table 1.

Since $\mathbf{L}_{S}^{2}$ contains redundant information, the ML-estimates $\hat{\delta}=\left(\hat{\delta}_{0} \hat{\delta}_{1} \ldots \hat{\delta}_{5}\right)^{T}$ in the first column of Table 1 are not reliable. Indeed the difference from the estimates in the second column is striking and illustrates the necessity of eliminating circular routes in SSAD models and other spatial models [see Blommestein (1985b) for a general discussion].

\section{A NEW ALGORITHM FOR THE ELIMINATION OF REDUNDANT PATHS IN SPATIAL LAG OPERATORS}

Ross and Harary (1952) present a very cumbersome procedure for the elimination of redundant paths. The procedure yields separate, non-recursive formulas. The complexity of these formulas increases progressively with increasing values of the power $p$ in $\mathbf{L}_{S}^{p}$ (see Appendix A for details). Much simpler, recursive procedures will be introduced in this paper.

Consider an $(R \times R)$ matrix $\mathbf{L}_{S}$. Non-triangular $\mathbf{L}_{S}^{p}$ will in general contain redundancies. A typical element from the matrix $\mathbf{L}_{S}^{2}$ can be written as

$$
l_{i j}^{2}=\sum_{k=1}^{R} l_{i k} l_{k j}
$$

A redundant path occurs when $i=j$ or $j=k$. To eliminate these paths, it is sufficient to add a restriction to (4.1), namely

$$
w_{i j}^{2}=\sum_{k=1}^{R} l_{i k} l_{k j} \quad[\text { with (i) } i \neq j, \text { (ii) } i \neq k \text {, (iii) } j \neq k]
$$

TABLE 1: Estimated Versions of Model (3.1)

\begin{tabular}{ccc}
\hline & With Redundant Paths & Without Redundant Paths \\
\hline$\delta_{0}$ & -17.32 & -13.39 \\
$\delta_{1}$ & +0.0008 & +0.0027 \\
$\delta_{2}$ & -0.0067 & -0.0034 \\
$\delta_{3}$ & +0.11 & +0.0049 \\
$\delta_{4}$ & +0.59 & +0.46 \\
$\delta_{5}$ & -0.11 & +0.04
\end{tabular}

Note: Uses the ML-search procedure proposed by Cliff and Ord (1981) and generalized by Blommestein (1983). The derivation of the asymptotic variance-covariance matrix is given in Blommestein (1985b). 
In this way redundancies are eliminated for $p=2$. In matrices raised to some arbitrary power $p(p>2)$, redundancies can be eliminated in a similar way

$$
w_{i j}^{p}=\sum_{k_{p-1}=1}^{R}\left(l_{i k_{p-1}} \sum_{k_{p-2}=1}^{R}\left(l_{k_{p-1} k_{p-2}}\left(\cdots \sum_{k_{1}=1}^{R}\left(l_{k_{2} k_{1}} l_{k_{1} j}\right) \cdots\right)\right)\right)
$$

with (i) $i \neq j$,

(ii) $i \neq k_{m}$, with $m=1, \ldots,(p-1)$,

(iii) $j \neq k_{m}$, with $m=1, \ldots,(p-1)$,

(iv) $k_{m} \neq k_{n}$, with $m, n=1, \ldots,(p-1)$ and $m \neq n$.

In terms of a recursion formula

$$
w_{i j}^{p}=\sum_{k_{p-1}=1}^{R}\left(l_{i k_{p-1}} w_{k_{p-1} j}^{p-1}\right)
$$

with (i) $i \neq j$,

(ii) $i \neq k_{m}$, with $m=1, \ldots,(p-1)$,

(iii) $j \neq k_{m}$, with $m=1, \ldots,(p-1)$,

(iv) $k_{m} \neq k_{n}$, with $m, n=1, \ldots,(p-1)$ and $m \neq n$.

Equation (4.4) presented here is a simple recursion formula, which can easily be translated into a computer algorithm. The computation time of (4.4) can be significantly reduced in some cases if one uses a priori knowledge about the structure of the spatial lag operator $\mathbf{L}_{S}$. For example,

(i) If $\mathbf{L}_{S}$ is triangular, no circular routes will occur for any $p$ so that $\mathbf{L}_{S}^{p}$ can be computed just by raising $\mathbf{L}_{S}$ to the $p$ th power (Blommestein, 1985a).

(ii) When $\mathbf{L}_{S}$ consists of relatively few non-zero elements (a so-called sparse matrix), the computation time can be reduced by testing for zero values in $\mathbf{L}_{S}$. Appendix $\mathrm{C}$ contains an algorithm implemented in PASCAL for this case.

(iii) if $p \geq R$ ( $R$ is the dimension of the spatial lag operator $\left.\mathbf{L}_{S}\right)$ then $\mathbf{L}_{S}^{p}=0$. In other words: in any $p$-step path $(p \geq R)$ a circular route must have occurred or otherwise there is no $p$ th order connection between the two vertices.

\section{IMPLEMENTATION AND RELATIVE COMPUTATION SPEED OF ALGORITHMS: THEORETICAL AND EMPIRICAL RESULTS}

The computation speed of an algorithm largely depends on the complexity of the algorithm. The complexity of the algorithm proposed by Ross and Harary (hereafter, $\mathrm{R} \& \mathrm{H}$ ) and our (hereafter, $\mathrm{B} \& \mathrm{~K}$ ) algorithm will be expressed in terms of number of multiplications, number of additions and number of comparisons that must be executed by a computer. The times consumed by program flow instructions (such as JUMP) and other overhead instructions (PUSH, POP, etc.) are not taken into account in this paper.

Generally speaking, computers use different computation times for multiplication, addition and comparison, and use more time to perform a difficult multiplication (like $3.1415927 \times 2.7182818$ ) than a simple one (like $1.0 \times 0.0$ ). The same is true for additions and sometimes for comparisons. Furthermore, floating point arithmetic requires more computation time than integer arithmetic. Let us assume that for some arbitrary computer system the average time needed for a multiplica- 
tion is $t_{m}$, for an addition is $t_{a}$, for comparison is $t_{c}$ and for a boolean comparison is $t_{b c}$.

First consider square matrix multiplication. We shall express the required time in terms of $t_{m}$ and $t_{a}$, assuming that no comparisons are made while multiplying matrices (the latter is actually not the case, but the total time needed for comparisons appears to be negligible in comparison with the time needed for the entire matrix multiplication).

A general $(R \times R)$ matrix $\mathbf{Q}$ consisting of typical elements $q_{i j}$ is multiplied by itself. The computation time needed for one typical element $q_{i j}^{2}$ is $R t_{m}+(R-1) t_{\alpha}$, so that the computation time required for the entire matrix $\mathbf{Q}^{2}$ is

$$
t_{M}=R^{2}\left[R t_{m}+(R-1) t_{a}\right]
$$

\section{Time Consumed by the $R \& H$ Algorithm}

We consider more closely the complexity of the procedure for the elimination of redundant paths as proposed by Ross and Harary (Appendix A and Ross and Harary, 1952). Separate (i.e., non-recursive) formulas are derived for the elimination of redundant paths up to six-step paths (raising the matrix to the sixth power). [The general symbolic representation of their formulas is given in Appendix A, Equation (A.4). Note that (A.4) is in non-computational form and that in this formula the term $\mathbf{C}^{P}$ must be derived for every $p$ separately.] Ross and Harary derive formulas for $p=2, \ldots, 6$.

Consider $p=2$. Equation (A.4) can be rewritten in computational form as

$$
\mathbf{W}^{2}=\mathbf{L}^{2}-d\left(\mathbf{L}^{2}\right)
$$

where $d$ represents diagonalization; that is, elimination of off-diagonal elements. The computation of $\mathbf{W}^{2}$ will cost

$$
\left.t_{W^{2}}=t_{M} \text { (to compute } \mathbf{L}^{2}\right)+R t_{a}(\text { to subtract the diagonal) }
$$

For $p=3$, first consider $\mathbf{C}^{3}$

$$
\mathbf{C}^{3}=\left[\mathbf{L} d\left(\mathbf{L}^{2}\right)+d\left(\mathbf{L}^{2}\right) \mathbf{L}\right]-\mathbf{S}
$$

where $\mathbf{S}=\mathbf{L} \times \mathbf{L}^{T}$ and $\times$ denotes the element-by-element matrix multiplication (Kronecker multiplication).

To compute $\mathbf{C}^{3}, \mathbf{L}^{2}$ must be computed first; then we must take the diagonal out of this matrix; the following step is to calculate two matrix products; next a matrix addition takes place; to compute $\mathbf{S}$, a Kronecker matrix multiplication is performed and finally another matrix addition (a subtraction is the same as an addition of the negative) yields $\mathbf{C}^{3}$. Hence the time to computer $\mathbf{C}^{3}$ is equal to

$$
t_{C^{3}}=3 t_{M}+2 t_{A}+t_{K}+t_{D}
$$

where

$$
\begin{aligned}
& t_{M}=R^{2}\left[R t_{m}+(R-1) t_{a}\right] \quad \text { (time needed for matrix multiplication); } \\
& t_{A}=R^{2} t_{a} \quad \text { (time needed for matrix addition); } \\
& t_{K}=R^{2} t_{m} \quad \text { (time needed for Kronecker matrix multiplication); } \\
& t_{D}=R t_{a} \quad \text { (time needed for a matrix diagonalization). }
\end{aligned}
$$


However, we are ultimately interested in the computation time of $\mathbf{W}^{3}$

$$
\mathbf{W}^{3}=\left(\mathbf{L}^{3}-\mathbf{C}^{3}\right)-d\left(\mathbf{L}^{3}-\mathbf{C}^{3}\right)
$$

Since $\mathbf{L}^{2}$ has already been computed, the time to compute $\mathbf{L}^{3}$ is the time needed for one matrix multiplication $\left(t_{M}\right)$. Furthermore one matrix subtraction and one matrix diagonalization (subtraction of the diagonal) have to be performed. Hence the time to compute $\mathbf{W}^{3}$ equals

$$
t_{W^{3}}=4 t_{M}+3 t_{A}+t_{K}+2 t_{D}
$$

In a similar fashion the computation time for $\mathbf{W}^{4}, \mathbf{W}^{5}$ and $\mathbf{W}^{6}$ can be derived.

$$
\begin{aligned}
& t_{W^{4}}=11 t_{M}+8 t_{A}+3 t_{K}+3 t_{D} \\
& t_{W^{5}} \approx 40 t_{M}+32 t_{A}+11 t_{K}+5 t_{D} \\
& t_{W^{6}} \approx 140 t_{M}+95 t_{A}+55 t_{K}+20 t_{D}
\end{aligned}
$$

These expressions are obtained by counting the multiplication signs, the addition signs, or other operators in the Ross and Harary formulas.

It can be seen that the first term in each of these expressions dominates the later terms. With increasing size of the matrix $(R)$ this becomes even more clear. Rewriting the expressions above by using $t_{m}$ and $t_{a}$, yields

$$
\begin{aligned}
t_{W^{2}} & \approx R^{3}\left(t_{m}+t_{a}\right) \\
t_{W^{3}} & \approx 4 R^{3}\left(t_{m}+t_{a}\right) \\
t_{W^{4}} & \approx 11 R^{3}\left(t_{m}+t_{a}\right) \\
t_{W^{5}} & \approx 40 R^{3}\left(t_{m}+t_{a}\right) \\
t_{W^{6}} & \approx 140 R^{3}\left(t_{m}+t_{a}\right)
\end{aligned}
$$

Time Consumed by the B\&K Algorithm

Two versions of the algorithm were developed. Version I is an implementation of Equation (4.4), while Version II exploits more fully the structure of the matrix by detecting zero values.

Consider Version I of the algorithm. Assume that we are dealing with a square non-triangular matrix $\mathbf{L}_{S}$ of dimension $(R \times R)$. The computation time for $\mathbf{W}^{P}$ for any $p\left(\in \mathbb{N}^{+}\right)$can be expressed in $t_{m}, t_{a}$ and $t_{b c}$

$$
\begin{aligned}
t_{W^{p}}=\left(t_{m}+t_{a}\right)\left(R^{2}-R\right) \sum_{n=2}^{p} \frac{(R-2) !}{(R-n-1) !} & \\
& \quad+t_{b c}\left[R^{2}+\left(R^{3}-R^{2}\right)\left(1+\sum_{n=3}^{p} \frac{(R-2) !}{(R-n) !}\right)\right]
\end{aligned}
$$

This can easily be derived from the structure of the algorithm [implementation of Equation (4.4)] which is presented in Appendix B.

Version II of our algorithm has the "overhead cost" of testing whether elements of the matrix are equal to zero (zero-detection), but it has the important 
advantage that no (expensive) multiplication has to be carried out if an element happens to be zero.

Definition 3. Let $R$ be the dimension of the square matrix $\mathbf{L}_{S}$. The fraction of nonzero off-diagonal elements is defined as

$$
f_{n z}=\frac{\text { Number of nonzero off-diagonal elements }}{(R-1) R}
$$

(Note that $0 \leq f_{n z} \leq 1$.)

Let the time needed to perform a comparison be $t_{c}$; then the computation time for $\mathbf{W}^{P}$ for any $p\left(\in \mathbb{N}^{+}\right)$using Version II of the B\&K algorithm, expressed in $t_{m}, t_{a}$, $t_{c}, t_{b c}$ and $f_{n z}$, is equal to

$$
\begin{aligned}
t_{W^{p}}= & \left(t_{m}+t_{a}\right)\left(R^{2}-R\right) \sum_{n=2}^{p}\left(\frac{(R-2) !}{(R-n-1) !}\right) f_{n z}^{p} \\
& +t_{b c}\left[R^{2}+\left(R^{3}-R^{2}\right)\left(1+\sum_{n=3}^{p}\left(\frac{(R-2) !}{(R-n) !} f_{n z}^{n-2}\right)\right)\right] \\
& +t_{c}\left(R^{2}-R\right) \sum_{n=2}^{p}\left(\frac{(R-2) !}{(R-n-1) !} f_{n z}^{n-2}\left(1+f_{n z}\right)\right)
\end{aligned}
$$

This can be derived from implementation of Version II of the B\&K algorithm as shown in Appendix C.

The advantage of Version II of the algorithm for sparse matrices is evident. It is readily verified that for $f_{n z}=1$, Version I is always faster than Version II; for $f_{n z}=$ 0 , Version II is always faster.

With the execution times $t_{m}, t_{a}, t_{c}$ and $t_{b c}$ given, the breakeven value for $f_{n z}$ can be computed for a combination of $R$ and $p$ by equating expressions (5.11) and (5.9). If $f_{n z}$ is larger than the break even value, B\&K Version I is faster; if it is smaller, $\mathrm{B} \& \mathrm{~K}$ Version II is faster.

It can easily be proved that B\&K Version II is faster than B\&K Version I for all combinations of $R$ and $p$ if

$$
f_{n z}<1-\frac{t_{c}}{t_{a}+t_{m}}
$$

Substituting the execution times listed in Table 2, Figure 2 shows results for $R=50$. For other values of $R$ a similar figure can be obtained. Not only the shape of the figure, but also the break-even values, are nearly the same as in Figure 2. For the computer we used in our tests, B\&K Version II is faster than B\&K Version I, for all values of $R$ and $p$, if $f_{n z}<0.58$.

\section{Empirical Results}

The R\&H and B\&K algorithms were implemented in PASCAL and executed on a Philips P2230 personal computer based on a 80286 Intel microprocessor chip. 
TABLE 2: Typical Integer Execution Times on a Philips P2230

$$
\begin{aligned}
t_{m}+t_{a} & =60 \mu \mathrm{s} \\
t_{c} & =25 \mu \mathrm{s} \\
t_{b c} & =14 \mu \mathrm{s}
\end{aligned}
$$

Typical execution times for $t_{m}+t_{a}, t_{c}$ and $t_{b c}$ are listed in Table 2 . The values for $t_{m}+t_{a}$ and $t_{c}$ are the execution times for operations on integer (or boolean) variables.

Since the $R \& H$ algorithm is only valid for boolean matrices, the three algorithms-R\&H, B\&K Version I and B\&K Version II-can only be compared when they are applied to boolean matrices. Matrices of dimension $(R \times R)$ $(2 \leq R \leq 100)$ were raised to powers $p(2 \leq p \leq 7)$, while redundant paths were eliminated by each of the three algorithms. Execution times were registered in milliseconds.

From Tables 3, 4 and 5 it can be seen that for large $R$ and for large $p$ the performance of the $\mathrm{B} \& \mathrm{~K}$ algorithms-in comparison with the $\mathrm{R} \& \mathrm{H}$ algorithmdecreases. Further, B\&K Version II performs better than B\&K Version I. In Appendix D, similar results are derived for B\&K Version I versus B\&K Version II, when both are applied to floating point matrices. The influence of $f_{n z}$ is demonstrated clearly.

Theoretical and empircal results roughly coincide. The reader can verify that substituting the values from Table 2 into Equations (5.8), (5.9) and (5.11) yields values very similar to those reported in Tables 3-5. Major deviations, either

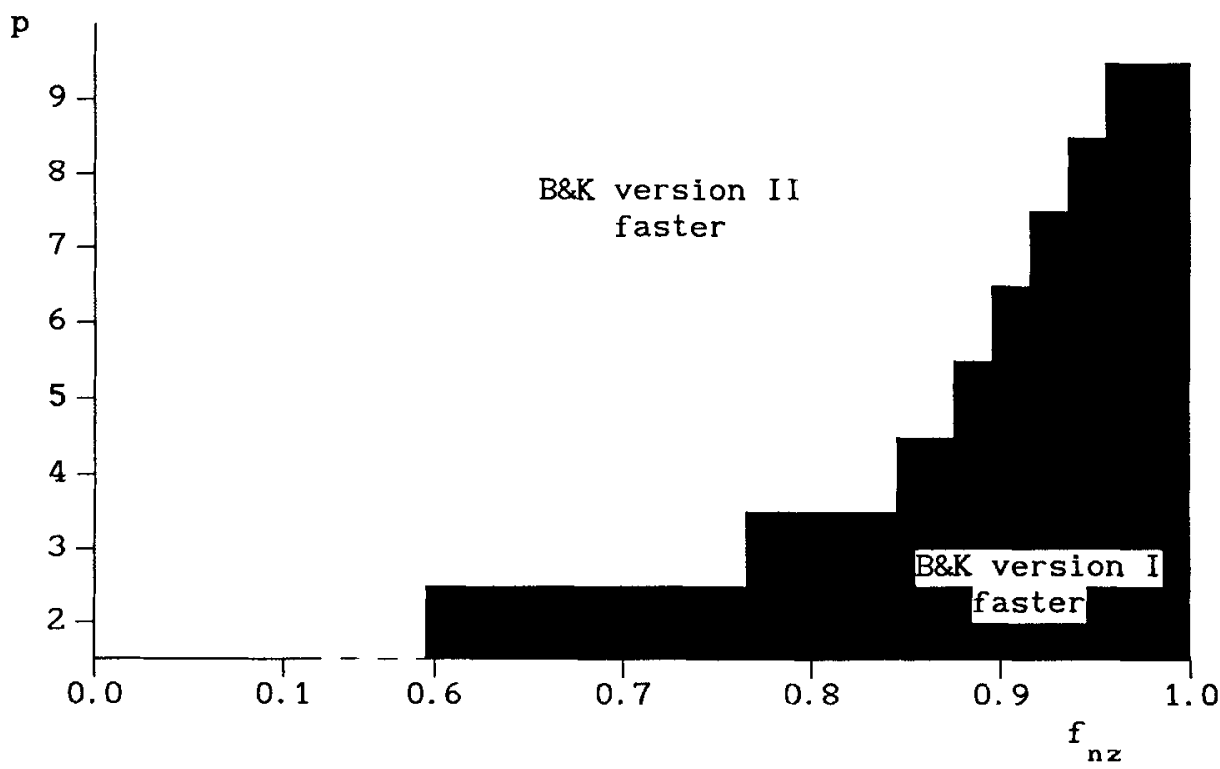

FIGURE 2: $f_{n z}$ versus $p$ for $R=50$. Note: In the black area, B\&K Version I is faster; in the white area B\&K Version II is faster. 
TABLE 3: R\&H Algorithm Execution Time For Raising an $(R \times R)$ Boolean Matrix to Power $p$ and Eliminating Redundant Paths

\begin{tabular}{|c|c|c|c|c|c|}
\hline & $p$ & 3 & 4 & 5 & 6 \\
\hline \multicolumn{6}{|l|}{$R$} \\
\hline 3 & 1 & & & & \\
\hline 5 & 4 & 15 & 40 & & \\
\hline 7 & 10 & 40 & 220 & 800 & 3000 \\
\hline 10 & 75 & 120 & 490 & 200 & 8000 \\
\hline 15 & 100 & 550 & 1600 & 8000 & 30000 \\
\hline 20 & 220 & 1300 & 3600 & 20000 & 70000 \\
\hline 30 & 830 & 4200 & 12000 & 60000 & 200000 \\
\hline 50 & 3800 & 19000 & 54000 & 300000 & 1000000 \\
\hline 70 & 10000 & 50000 & 140000 & 800000 & 3000000 \\
\hline 100 & 75000 & 140000 & 440000 & 2000000 & 8000000 \\
\hline
\end{tabular}

Note: Entries denote execution times in milliseconds. For $p=5,6$ the R\&H algorithm was not implemented; instead the results in (5.8) were used to approximate the computation times. Thus, entries in italics were not actually measured.

positive or negative, arise from the presence or absence of extra program flow and/or indexed memory-addressing instructions.

To conclude this section, it must be emphasized that the implementation of the $\mathrm{R} \& \mathrm{H}$ formulas for $p=5,6$ is a very time consuming job, and that the derivation of the $\mathrm{R} \& H$ formulas for $p>6$ is an extremely time consuming and tedious task. The implementation time and required work space of the $\mathrm{R} \& \mathrm{H}$ algorithm is not recorded and therefore a comparison with the B\&K algorithm solely based on (approximate) execution times is biased in favor of the R\&H algorithm. However, it seems evident that the non-recursive $\mathrm{R} \& \mathrm{H}$ algorithm is not very attractive to use in empirical work.

TABLE 4: B\&K's Version I Algorithm Execution Time for Raising an $(R \times R)$ Boolean Matrix to Power $p$ and Eliminating Redundant Paths

\begin{tabular}{|c|c|c|c|c|c|c|}
\hline & $p$ & 3 & 4 & 5 & 6 & 7 \\
\hline \multicolumn{7}{|l|}{$R$} \\
\hline 3 & 0 & & & & & \\
\hline 5 & 5 & 15 & 30 & & & \\
\hline 7 & 16 & 50 & 110 & 390 & 820 & \\
\hline 10 & 60 & 170 & 1100 & 6200 & 29000 & 300000 \\
\hline 15 & 110 & 880 & 11000 & 110000 & 300000 & 30000000 \\
\hline 20 & 160 & 3100 & 51000 & 800000 & 3000000 & $4 E 8$ \\
\hline 30 & 610 & 17000 & 440000 & 10000000 & $8 E 8$ & $2 E 10$ \\
\hline 50 & 2800 & 130000 & 7000000 & $4 E 8$ & $4 E 10$ & $2 E 12$ \\
\hline 70 & 80000 & 350000 & $5 E 7$ & $6 E 9$ & $5 E 11$ & $3 E 13$ \\
\hline 100 & 24000 & 2400000 & $3 E 8$ & $5 E 10$ & $6 E 12$ & $6 E 14$ \\
\hline
\end{tabular}

Note: Entries denote execution times in milliseconds. The entries in italics were approximated using Equation 5.9. 
TABLE 5: B\&K's Version II Algorithm Execution Time for Raising an $(R \times R)$ Boolean Matrix to Power $p$ and Eliminating Redundant Paths. $f_{n z}=0.4$

\begin{tabular}{rrrrrrr}
\hline \hline & $p$ & 2 & 3 & 4 & 5 & \multicolumn{1}{c}{6} \\
\hline \multicolumn{1}{r}{$R$} & & & & & & \\
3 & 0 & & & & & \\
5 & 1 & 3 & 4 & & & \\
7 & 5 & 36 & 21 & 30 & 40 & \\
10 & 15 & 110 & 220 & 600 & 1000 & 2800 \\
15 & 50 & 440 & 1900 & 7400 & 32000 & 180000 \\
20 & 110 & 1300 & 8600 & 49000 & 800000 & 4000000 \\
30 & 550 & 6100 & 72000 & 2000000 & 20000000 & $2 E 8$ \\
50 & 2600 & 50000 & 3000000 & 5000000 & $8 E 8$ & $1 E 10$ \\
70 & 6500 & 500000 & 10000000 & $4 E 8$ & $1 E 10$ & $2 E 11$ \\
100 & 19000 & 2000000 & 90000000 & $3 E 9$ & $1 E 11$ & $5 E 12$ \\
\hline
\end{tabular}

Note: Entries denote execution times in milliseconds. The entries in italics were approximated using Equation 5.11.

\section{FINAL REMARKS}

In this paper we studied the problem of redundant paths in spatial lag operators. In addition, we discussed two procedures for the elimination of such redundant paths.

The procedure developed by Blommestein and Koper has a much simpler structure than Ross and Harary's procedure, because it is recursive. R\&H's formulas must be derived for each $p$ separately, which for large $p(>6)$ must be considered a tedious job. Moreover, B\&K's procedure is suited for all types of matrices, while R\&K's procedure is only suited for boolean matrices. Furthermore, B\&K's Version II is superior for sparse matrices of all types.

\section{REFERENCES}

Blommestein, Hans J. 1983. "Reanalysis of an Irregular Lattice," in R. Henn et al. (eds.), Methods of Operations Research, 46, Königstein, Athenäum Printers, pp. 1-14.

- 1984. "Mathematical Properties and Econometric Applications of Spatial Lag Operators," paper presented at the 23rd Western Regional Science Conference, February 22-25, Monterey, USA.

_. 1985a. "Elimination of Circular Routes in Spatial Dynamic Regression Equations," Regional Science and Urban Economics, 15, 121-130.

—. 1985b. "Eliminative Econometrics," unpublished Ph.D. dissertation, Department of Economics, University of Amsterdam (VU).

Blommestein, Hans J. and Nick A. M. Koper. 1984. "Analysis of Redundant Path in Spatial Dynamic Systems," in R. Henn et al. (eds.), Methods of Operations Research, 52, Königstein, Athenäum Printers, pp. 573-582.

Ross, Ian C. and Frank Harary. 1952. "On the Determination of Redundancies in Sociometric Chains," Psychometrika, 17, 195-208.

APPENDIX A: Summary and Evaluation of Ross and Harary's Procedure for the Elimination of Redundant Paths

The procedure presented by Ross and Harary (1952) is very complicated. Their procedure leads to separate, non-recursive formulas for the elimination of all orders of redundancies. Furthermore, their formulas are strictly speaking only 
valid for boolean matrices, although their method could possibly be adapted for more general types of matrices. This appendix summarizes R\&H's procedure. Refer to Ross and Harary (1952) for an extensive discussion. Example 1 from Section 2 is used to illustrate the $R \& H$ algorithm.

Consider an economic agent $A_{i}$ and an economic agent $A_{j}$. A path from $A_{i}$ to $A_{j}$ is a chain of steps starting with $A_{i}$ and ending with $A_{j}$. For example, $A_{i} \rightarrow A_{k} \rightarrow A_{j}$ is a two-step path. The elements $w_{i j}^{p}$ of matrix $\mathbf{L}_{s}^{p}$ indicate how many $p$-step paths there exist from $A_{i}$ to $A_{j}$.

Ross and Harary define a matrix $\mathbf{C}^{p}$ consisting of elements $c_{i j}^{p}$. The elements $c_{i j}^{p}$ indicate how many $p$-step redundant paths there are from $A_{i}$ to $A_{j}$, with $i \neq j$. Ross and Harary split the integer value $p$ into three other integers, $p_{1}, p_{2}, p_{3}$ in such a way that

$$
p_{1}+p_{2}+p_{3}=p \text { and } p_{1}, p_{2}, p_{3} \geq 0, p_{1}+p_{3}<0, p_{2}>1
$$

Such a combination of $p_{1}, p_{2}, p_{3}$ is called a partition of $p$. Assume that $p_{1}, p_{2}, p_{3}$ is a partition of $p$. A redundant path from $A_{i}$ to $A_{j}$ satisfies this partition if

$p_{1}=$ the number of steps from the initial agent $A_{i}$ to one of the places in which a repeated agent occurs other than the last appearance.

$p_{2}=$ the number of steps from this appearance of the repeated agent to a later appearance.

$p_{3}=$ the number of steps from this second appearance to the terminal or final agent $A_{j}$.

Example

$$
A_{i} \rightarrow A_{k} \rightarrow A_{l} \rightarrow A_{m} \rightarrow A_{k} \rightarrow A_{n} \rightarrow A_{j}
$$

The repeated agent is $A_{k}$, so $p_{1}=1, p_{2}=3$ and $p_{3}=2$. This is called a 132 partition. However, it is possible that some arbitrary path satisfies more than one partition.

Example

$$
A_{i} \rightarrow A_{j} \rightarrow A_{i} \rightarrow A_{j}
$$

Consider $A_{i}$ as a repeated agent. Hence $p_{1}=0, p_{2}=2, p_{3}=1$ (a 021 partition). But we can also consider $A_{j}$ as a repeated agent. Then $\mathrm{p}_{1}=1, \mathrm{p}_{2}=2, \mathrm{p}_{3}=0$ (a 120 partition).

The maximum number of partitions a redundant $p$-step path can possibly satisfy is

$$
u=2 K(\operatorname{Round}(p / 2), 2)
$$

in which

$$
K(n, m)=\frac{n !}{m !(n-m) !}
$$

and Round( ) denotes the nearest integer such that

$$
\operatorname{Round}(0.5)=1, \operatorname{Round}(1)=1, \operatorname{Round}(1.5)=2, \quad \text { etc. }
$$


The total number of partitions of $p$ is

$$
q=K(p, 2)-1
$$

For each $i, j$ and each $p$, Ross and Harary derive the number of redundant paths $c_{i j}^{P}$.

Assume that

(i) $f_{1}, f_{2}, \ldots, f_{q}$ is the set of all partitions of $p$; remember that $q=K(p, 2)-1$.

(ii) $b\left[x_{1}, x_{2}, \ldots, x_{t}\right]$ is the number of paths that satisfy $f_{x_{1}}$ and $f_{x_{2}}$ and $\ldots$ and $f_{x_{t}}$, while $1 \leq x_{p} \leq q$ and $x_{i} \neq x_{j}$.

(A.1) (iii) $B_{t}=\sum_{x_{t}=x_{t-1}+1} \ldots \sum_{x_{2}=x_{1}+1} \sum_{x_{1}=1} b\left[x_{1}, x_{2}, \ldots, x_{t}\right]$

for each $t$, with $1 \leq t \leq u$. Define $c_{i j}^{p}$ as follows:

$$
c_{i j}^{p}=\sum_{t=1}^{u}\left[(-1)^{(t+1)} B_{t}\right]
$$

Let $\mathbf{C}_{t}^{p}$ be the matrix whose $i, j$ entry is $\mathrm{B}_{\mathrm{t}}$; then

$$
C^{p}=\sum_{t=1}^{u}\left[(-1)^{(t+1)} C_{t}^{p}\right]
$$

To eliminate all redundancies from $\mathbf{L}_{S}^{p}$, the final step is to use the diagonalization operator

$$
\mathbf{W}^{p}=\left(\mathbf{L}^{p}-\mathbf{C}^{p}\right)-d\left(\mathbf{L}^{p}-\mathbf{C}^{p}\right)
$$

in which

$\mathbf{W}^{p}$ is the matrix $\mathbf{L}$ raised to the power $p$ while redundant paths were eliminated;

$\mathbf{L}$ is the original matrix;

$p$ is the power to which $\mathbf{L}$ is raised;

$\mathbf{C}^{p}$ is the matrix containing all $p$-step redundant paths except for the diagonal, and;

$d$ is the diagonalization operator.

Formulas for the elimination of redundant paths were derived by Ross and Harary for $p$-values up to six. A major drawback of the Ross and Harary formulas is that they are very difficult to derive (especially for large $p$ ). Moreover, their procedure yields non-recursive and very complex formulas. $\mathrm{R} \& \mathrm{H}$ 's formula (6) for the elimination if $p=$ six-step redundant paths occupies two printed pages in their article!

Another point of criticism is that strictly speaking the formulas are only valid for boolean matrices. For example, using Ross and Harary's formula (4) (on page 201) for eliminating redundant paths from matrices raised to the fourth power, using the matrix representation $\mathbf{L}_{S}$. Raising $\mathbf{L}_{S}$ to the fourth power yields redundant paths. In case the matrix $\mathbf{L}_{S}$ contains elements not equal to values 0 or 1 , then the formula does not yield the desired result. For example, replace all 1's in 
$\mathbf{L}_{S}$ on page 94 by the value 2

$$
\mathbf{L}_{S}=\left[\begin{array}{llllll}
0 & 2 & 0 & 2 & 2 & 0 \\
2 & 0 & 0 & 2 & 2 & 0 \\
0 & 2 & 0 & 0 & 2 & 2 \\
2 & 0 & 2 & 0 & 2 & 0 \\
2 & 2 & 0 & 2 & 0 & 0 \\
0 & 2 & 2 & 0 & 2 & 0
\end{array}\right]
$$

Eliminating redundant paths from the matrix $\mathbf{L}_{S}^{4}$, using the procedure proposed by Ross and Harary, yields

$$
\mathbf{L}_{S}^{4}=\left[\begin{array}{rrrrrr}
0 & 16 & 24 & -32 & 0 & 32 \\
-16 & 0 & 32 & -56 & 0 & 32 \\
96 & 8 & 0 & 120 & 40 & 0 \\
32 & 0 & -16 & 0 & 0 & -8 \\
-32 & 0 & 24 & -40 & 0 & 32 \\
96 & 32 & 64 & 120 & 64 & 0
\end{array}\right]
$$

Note that negative values in this matrix are not defined and therefore not allowed. 
APPENDIX B: Blommestein \& Koper's Algorithm Version I

procedure BKversionI (var 1: matrix; p: integer; var w: matrix);

var $i, j$ : integer;

NOTinpath: array $[1 . \mathrm{R}]$ of boolean;

function computew( $i, j, p$ : integer ): real;

var wij: real;

begin

k: integer;

wij: $=0$;

if $p=2$ then

begin

for $k:=1$ to $R$ do

if NOTinpath[k] then

end

wi $j:=w i j+1[i, k]^{*} 1[k, j]$;

else

begin

for $k:=1$ to $R$ do

if NOTinpath[k] then

begin

NOT inpath $[k]:=$ false;

wi j: $=w i j+1[i, k] *$ computeW $(k, j, p-1)$;

NOTinpath $[\mathrm{k}]:=$ true;

end;

end;

computew: $=w i j$;

end;

begin

for $i:=1$ to $R$ do NOTinpath[i]:=true;

for $i:=1$ to $R$ do

for $j:=1$ to $R$ do

if $i<>j$ then

begin

NOTinpath $[i]:=f a]$ se; NOTinpath $[j]:=$ false;

$w[i, j]:=\operatorname{computew}(i, j, p)$;

NOT inpath $[i]:=\operatorname{true;}$ NOTinpath $[j]:=\operatorname{true;~}$

end

else

end;

$w[i, j]:=0$; 
APPENDIX C: Blommestein \& Koper's Algorithm Version II

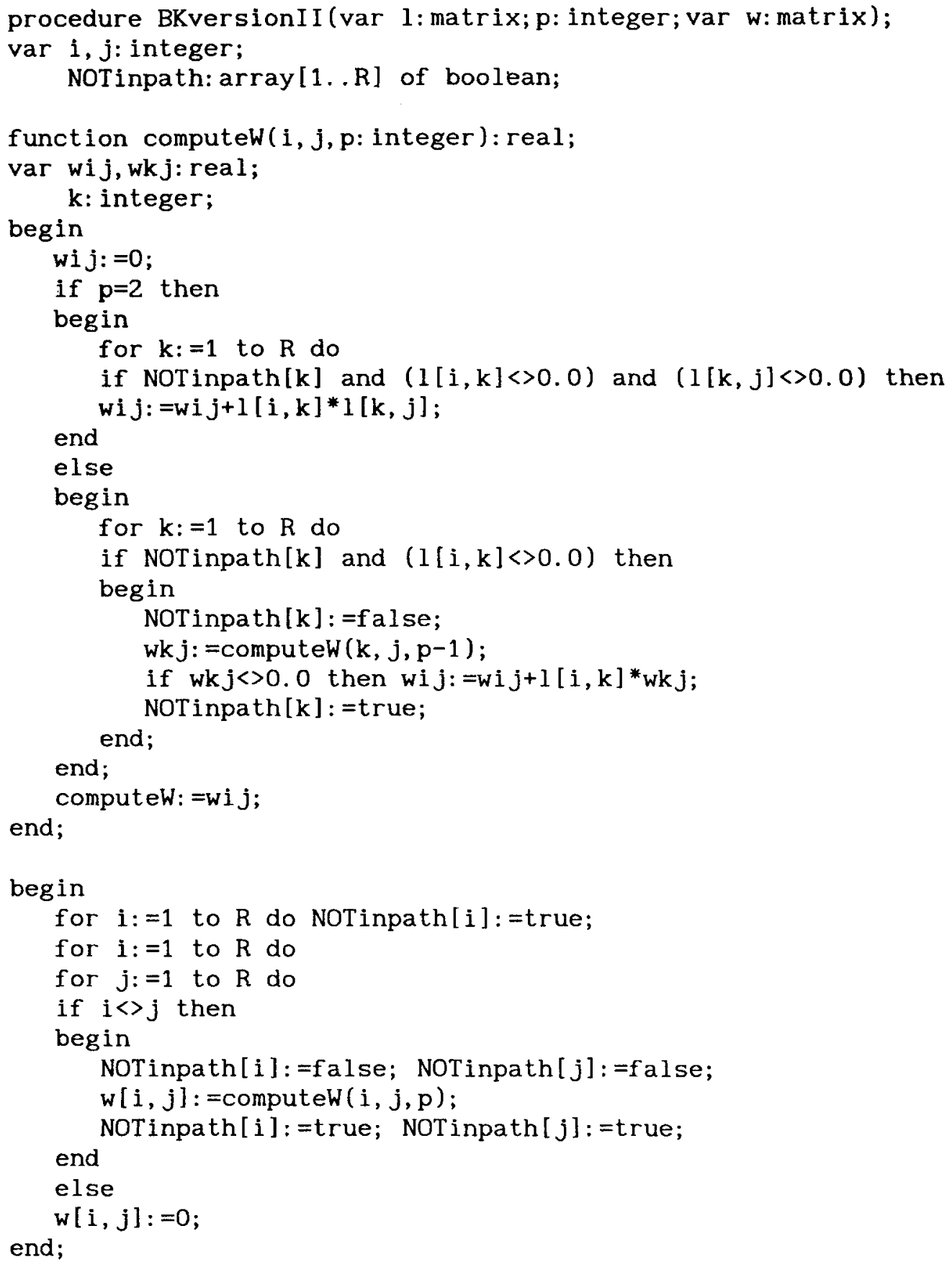


APPENDIX D: Additional Results on the Performance of the B\&K Algorithms

The B\&K algorithms are-unlike the $\mathrm{R} \& \mathrm{H}$ algorithm - valid for all types of matrices. Floating point matrices of dimension $R(3 \leq R \leq 50)$ with several $f_{n z}$ values $\left(0 \leq f_{n z} \leq 1\right)$ were supplied to the B\&K Version I and Version II algorithm. The matrices were raised to powers $p(2 \leq p \leq 6)$, while redundant paths were eliminated. Execution times were registered in milliseconds. Table D.1 presents the typical execution times $t_{a}+t_{m}$ and $t_{c}$ of elementary floating point operations. Notice that $t_{b c}$ is unchanged with respect to Table 5.1.

Table D.2 shows the execution times for B\&K Version I. Tables D.3 up to D.7 display the results for $\mathrm{B} \& \mathrm{~K}$ Version II with $f_{n z}=1,0.7,0.4,0.2$ and 0.1 .

TABLE D.1: Typical Floating Execution Times on a Philips P2230

$$
\begin{aligned}
t_{m}+t_{a} & =500 \mu \mathrm{s} \\
t_{c} & =90 \mu \mathrm{s} \\
t_{b c} & =14 \mu \mathrm{s}
\end{aligned}
$$

\begin{tabular}{|c|c|c|c|c|c|}
\hline & $p$ & 3 & 4 & 5 & 6 \\
\hline \multicolumn{6}{|l|}{$R$} \\
\hline 3 & 1 & & & & \\
\hline 5 & 8 & 25 & 40 & & \\
\hline 7 & 25 & 140 & 460 & 1200 & 1900 \\
\hline 10 & 90 & 750 & 4600 & 24000 & 1000000 \\
\hline 15 & 350 & 4500 & 50000 & 500000 & \\
\hline 20 & 900 & 16000 & 25000 & & \\
\hline 30 & 3000 & 90000 & & & \\
\hline 50 & 15000 & 750000 & & & \\
\hline
\end{tabular}

TABLE D.2: B\&K's Version I Algorithm Execution Time for Raising an $(R \times R)$ Real Matrix to Power $p$ and Eliminating Redundant Paths

Note: Entries denote execution times in milliseconds.

TABLE D.3: B\&K's Version II Algorithm Execution Time for Raising an $(R \times R)$ Real Matrix to Power $p$ and Eliminating Redundant Paths $-f_{n z}=1$

\begin{tabular}{rrrrrr}
\hline \hline$R$ & 2 & 3 & 4 & 5 & 6 \\
\hline \multicolumn{1}{l}{$r$} & & & & & \\
5 & 1 & & & & \\
7 & 10 & 30 & 55 & 1500 & 2500 \\
10 & 35 & 180 & 650 & 33000 & 140000 \\
15 & 125 & 1000 & 6500 & 700000 & \\
20 & 480 & 6000 & 68000 & & \\
30 & 1100 & 21000 & 350000 & & \\
50 & 4000 & 120000 & & & \\
\hline
\end{tabular}

Note: See Note to Table D.2. 
TABLE D.4: B\&K's Version II Algorithm Execution Time for Raising an $(R \times R)$ Real Matrix to Power $p$ and Eliminating Redundant Paths- $f_{n z}=0.7$

\begin{tabular}{|c|c|c|c|c|c|}
\hline & $p$ & 3 & 4 & 5 & 6 \\
\hline \multicolumn{6}{|l|}{$R$} \\
\hline 3 & 1 & & & & \\
\hline 5 & 6 & 15 & 19 & & \\
\hline 7 & 22 & 80 & 200 & 350 & 460 \\
\hline 10 & 75 & 420 & 1900 & 7100 & 22000 \\
\hline 15 & 280 & 2600 & 20000 & 150000 & \\
\hline 20 & 710 & 9000 & 100000 & & \\
\hline 30 & 2500 & 50000 & & & \\
\hline 50 & 12000 & 410000 & & & \\
\hline
\end{tabular}

Note: See Note to Table D.2.

TABLE D.5: B\&K's Version II Algorithm Execution Time for Raising an $(R \times R)$ Real Matrix to Power $p$ and Eliminating Redundant Paths- $f_{n z}=0.4$

\begin{tabular}{rrrrrr}
\hline \hline & $p$ & 2 & 3 & 4 & \multicolumn{1}{c}{5} \\
\hline$R$ & & & & & 6 \\
3 & 0 & & 6 & & \\
5 & 4 & 68 & 48 & 62 & 1700 \\
7 & 12 & 150 & 400 & 16000 & 61000 \\
10 & 40 & 850 & 19000 & 120000 & \\
15 & 160 & 2800 & 160000 & & \\
20 & 380 & 16000 & & & \\
30 & 1300 & 130000 & & & \\
50 & 6500 & & &
\end{tabular}

Note: See Note to Table D.2.

TABLE D.6: B\&K's Version II Algorithm Execution Time for Raising an $(R \times R)$ Real Matrix to Power $p$ and Eliminating Redundant Paths- $f_{n z}=0.2$

\begin{tabular}{|c|c|c|c|c|c|}
\hline & $p$ & 3 & 4 & 5 & 6 \\
\hline \multicolumn{6}{|l|}{$R$} \\
\hline 3 & 0 & & & & \\
\hline 5 & 2 & 3 & 3 & & \\
\hline 7 & 8 & 14 & 17 & 18 & 18 \\
\hline 10 & 26 & 61 & 100 & 140 & 180 \\
\hline 15 & 99 & 330 & 820 & 1800 & 3700 \\
\hline 20 & 250 & 1100 & 3600 & 12000 & 33000 \\
\hline 30 & 850 & 5500 & 30000 & 150000 & \\
\hline 50 & 4200 & 43000 & 400000 & & \\
\hline
\end{tabular}

Note: See Note to Table D.2.

From Tables D.2-D.7 it can be concluded that in practice: (1) Version II of the B\&K algorithm performs better than Version I, (2) the superior performance of Version II versus Version I increases with decreasing values of $f_{n z}$, and (3) only for $f_{n z}=1$ Version I is always faster. 
TABLE D.7: B\&K's Version II Algorithm Execution Time for Raising an $(R \times R)$ Real Matrix to Power $p$ and Eliminating Redundant Paths- $f_{n z}=0.1$

\begin{tabular}{rrrrrr}
\hline \hline$R$ & $p$ & 3 & 4 & 5 & \multicolumn{1}{c}{6} \\
\hline \multicolumn{1}{c}{$r$} & 2 & & & & \\
3 & 0 & 2 & 2 & & \\
5 & 2 & 9 & 10 & 10 & 10 \\
7 & 7 & 37 & 46 & 51 & 55 \\
10 & 22 & 190 & 290 & 390 & 490 \\
15 & 83 & 550 & 1100 & 1900 & 3100 \\
20 & 210 & 2600 & 7700 & 20000 & 51000 \\
30 & 730 & 95000 & 430000 & \\
50 & 3500 & 2000 & & & \\
\hline
\end{tabular}

Note: See Note to Table D.2. 